\title{
Hydrostatic pressure effects on a hydrated lipid inverse micellar Fd3m cubic phase
}

\author{
A. I. I. Tyler, ${ }^{a}$ G. C. Shearman, ${ }^{a}$ N. J. Brooks, ${ }^{a}$ H. Delacroix, ${ }^{b}$ R. V. Law, ${ }^{a}{ }^{a}$ R. H. Templer, ${ }^{a}$ O. Ces, ${ }^{a}$ \\ and J. M. Seddon* ${ }^{a}$ \\ ${ }_{5}$ Received (in $\left.X X X, X X X\right) X$ th $X X X X X X X X X 200 X$, Accepted Xth $X X X X X X X X X 200 X$ \\ First published on the web Xth $X X X X X X X X X 200 X$ \\ DOI: $10.1039 / b 000000 x$
}

10
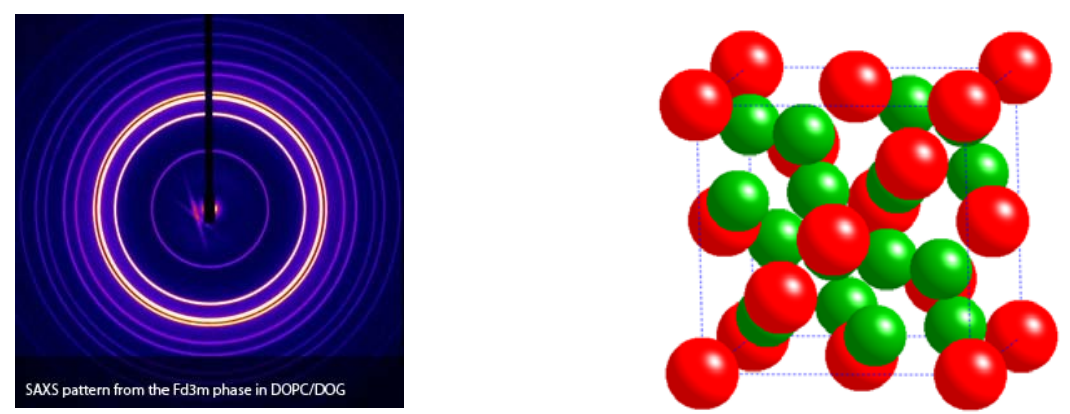

${ }_{20}$ Graphical contents entry. The effect of hydrostatic pressure on an inverse micelllar Fd3m cubic phase is studied by small-angle synchrotron diffraction.

Over a range of hydration, unsaturated diacylglycerol / phosphatidylcholine mixtures adopt an 25 inverse micellar cubic phase, of crystallographic space group Fd3m. In this study hydrated DOPC: DOG mixtures with a molar ratio close to 1:2 were examined as a function of hydrostatic pressure, using synchrotron $\mathrm{x}$-ray diffraction. The small-angle diffraction pattern at atmospheric pressure was used to calculate 2-D sections through the electron density map. Pressure initially has very little effect on the structure of the Fd3m cubic phase, in contrast to its effect on hydrated inverse 30 bicontinuous cubic phases. At close to $2 \mathrm{kbar}$, a sharp transition occurs from the Fd $3 \mathrm{~m}$ phase to a pair of coexisting phases, an inverse hexagonal $\mathrm{H}_{\mathrm{II}}$ phase plus an (ordered) lamellar phase. Upon increasing the pressure to $3 \mathrm{kbar}$, a further sharp transition occurs from the $\mathrm{H}_{\mathrm{II}}$ phase to a (fluid) lamellar phase, in coexistence with the ordered lamellar phase. These transitions are fully reversible, but show hysteresis. Remarkably, the lattice parameter of the Fd3m phase is practically 35 independent of pressure. These results show that these two lipids are miscible at low pressure, adopting a single lyotropic phase $(\mathrm{Fd} 3 \mathrm{~m})$; they then become immiscible above a critical pressure, phase separating into DOPC-rich and DOG-rich phases.

\section{Introduction}

Lipids are amphiphilic molecules which can self-assemble 40 into a wide range of liquid-crystalline phases in aqueous solution, depending on the balance between the polar headgroup packing, and the hydrocarbon chain packing. Lipids with small, weakly polar headgroups and multiple, long alkyl chains have a tendency to adopt inverse phases 45 with increasing temperature. In such phases, the polarnonpolar interface adopts a negative mean curvature, in the sense that the interface curves towards the water and away from the hydrocarbon chain region. Starting from the flat fluid lipid bilayer (zero mean curvature), which forms the basis of 50 the structure of nearly all biological membranes, first inverse bicontinuous cubic, then inverse hexagonal $\mathrm{H}_{\text {II }}$ phases may be observed upon varying any parameter that tends to favour inverse curvature (such as increasing temperature, increasing number or degree of unsaturation of the lipid hydrocarbon ${ }_{55}$ chains, etc) ${ }^{1}$. In extreme cases, some very weakly amphiphilic lipids may adopt even more highly negatively-curved phases, such as the inverse micellar cubic phase of spacegroup Fd3m (Fig.1). 


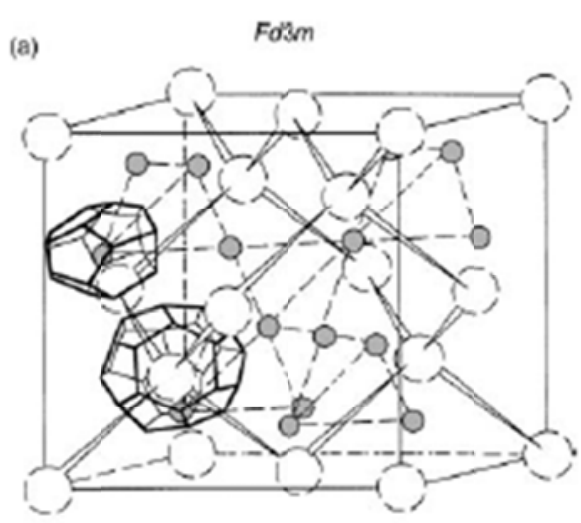

(b)

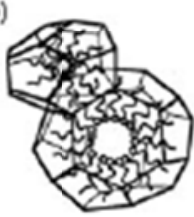

Fig.1 Schematic structure of the lyotropic cubic phase of spacegroup Fd3m: (a) arrangement of the inverse micelles within the unit cell (origin choice 1 at $\overline{4} 3 \mathrm{~m}$ - see International Tables for Crystallography); (b) the connection between the hexakaidecahedral and the dodecahedral volumes occupied by the two types of inverse micelle. Taken from ref. 2.

For pure phospholipids in water, such ordered inverse discontinuous phases have not so far been found; however, by 10 addition of a weakly polar amphiphile such as protonated fatty acids, fatty alcohols, or diacylglycerols (DAG) to phospholipids such as phosphatidylcholine (PC) (Fig. 2a), the preferred interfacial curvature can be tuned to be so strongly negative that ordered inverse micellar phases are observed ${ }^{2-5}$.

15 Such molecules are soluble in phospholipids to high mole fractions $(\geq 0.67)$, above a certain temperature. It should be noted however that the $\mathrm{Fd} 3 \mathrm{~m}$ phase has been observed in certain single hydrated glycolipid systems such as the dialkylxylopyranosylglycerol/water

20 diphatanylglucosylglycerol/water systems ${ }^{6,7}$.

Another way of promoting the inverse micellar Fd3m phase to form is to reduce the packing frustration in the chain region by adding non-polar solutes (such as alkanes) to amphiphiles such as monoacylglycerols ${ }^{8-11}$. Also non-polar molecules such 25 as limonene $e^{8,12-14}$ can have the same effect.

Diacylglycerols (Fig. 2b) are of particular interest in biology, as they are involved in signalling pathways in cells. Diacylglycerols have a relatively simple chemical structure 30 compared to other membrane lipids and are found at relatively low concentrations in cell membranes under equilibrium conditions and because of this their association with a wide range of biological responses was originally questioned. It is now well established that they act as second messengers in

35 cell signalling, are key intermediates in the metabolism of many lipid moieties, they trigger translocation and subsequently activation of enzymes to the membrane and modulate the activity of many membrane proteins ${ }^{15}$.Diacylglycerols can be produced via multiple
90 pathways in the cell, such as by the action of the enzyme phospholipase. The resulting diacylglycerol remains in the membrane, activating the membrane-bound enzyme protein kinase $\mathrm{C}$, and perturbing the locall membrane structure in ways which are poorly understood. Moreover, their local and 95 transient $^{16}$ accumulation in membranes as well as their rapid diffusion within the membrane can result in local clusters of high DAG concentration to be accumulated which could lead to a change in the structure of the membrane, facilitating processes such as membrane fission and fusion.

55

a)

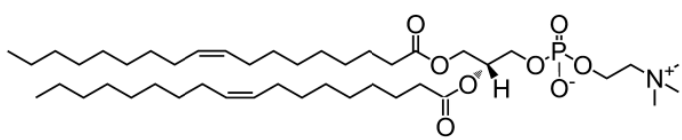

b)

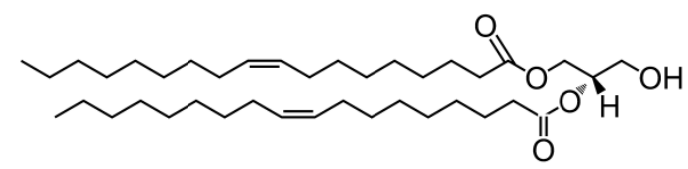

65 Fig.2 Chemical structures of (a) 1,2-dioleoyl-sn-glycero-3phosphatidylcholine(DOPC) and (b) 1,2-dioleoylglycerol (DOG).

The limiting hydration of the $\mathrm{Fd} 3 \mathrm{~m}$ cubic phase of DOPC/DOG has been determined to be $20-25 \mathrm{wt}^{\%}$ water $^{1}$, 75 with a lattice parameter at full hydration close to $150 \AA$.

We recently found that addition of cholesterol to a hydrated PC/DAG mixture can induce a novel 3-D hexagonal phase to form, of spacegroup $\mathrm{P} 6_{3} / \mathrm{mmc}$, whose structure consists of a hexagonal close packing of identical inverse micelles ${ }^{17}$.

${ }_{110}$ Ordered inverse micellar phases such as the Fd3m cubic phase have obvious potential in nanomedicine as vehicles for the slow release of water-soluble drugs; more generally, they could find applications as ordered arrays of isolated aqueous nanoreactors of uniform size, with aqueous volumes as small 115 as $30 \mathrm{~nm}^{3}$ ( $30 \times 10-^{24}$ litres). Recently they have been used to template mesocaged cubic Fd3m silica monoliths, which have been developed as carriers for optical chemosensing of waste water pollutants such as bismuth ions ${ }^{18}$.

Although many studies have focussed their attention on DAG 140 containing systems and the effiect of temperature on their phase behaviour ${ }^{2,5,19-25}$, few studies have investigated the effect of pressure. In the present study, the effect of pressure at different temperatures on the equilibrium phase behaviour of fully hydrated DOPC: DOG $\left(\mathrm{c}_{\mathrm{DOG}}=0.7\right)$ mixtures has been 145 investigated using synchrotron small angle $x$-ray scattering, allowing two dimensions in phase space to be explored. Pressure can provide information on phases that would not be possible through other variables, unless the chemical properties of the lipid species were altered ${ }^{26}$.The effect of 150 pressure on lipid phase behaviour tends to be opposite to the effect of temperature. Pressure promotes any chemical or 
physical change that results in a volume reduction ${ }^{27}$ and hence an increase in pressure tends to stabilise phases with low curvatures, since hydrocarbon chains occupy lower volumes as they become conformationally more ordered.

\section{Materials and Methods}

Dioleoyl-sn-glycerol (DOG) was purchased from Larodan Fine Chemicals (Malmo, Sweden) and 1,2-dioleoyl-snglycero-3-phosphocholine (DOPC) from Avanti Polar Lipids, 10 Inc. (Birmingham, AL.). All of the lipids had a purity $>99 \%$ and were used without further purification. Each lipid was freeze-dried individually and samples were prepared by mixing the desired amount of dry lipids and dissolving them in cyclohexane, with a few drops of methanol to aid 15 dissolution. The mixture was frozen in liquid nitrogen and lyophilised for at least 24 hours to remove the solvent, and excess water $(60 \mathrm{wt} \%)$ was then added to the sample. The sample used for the electron density map reconstruction had a water content of $19 \mathrm{wt} \%$. All water used in sample preparation 20 was de-ionised, HPLC grade. The sample was subjected to several freeze-thaw cycles, and a minimum of 5 pressure ( 1 to 2000 bar) cycles in order to achieve reproducibility and sample homogeneity.

X-ray experiments were carried out on the high brilliance 25 beamline ID02 at the European Synchrotron Radiation Facility (ESRF), using a high pressure cell, as previously described $^{28}$. The synchrotron X-ray beam was tuned to a wavelength of $0.7514 \AA(\mathrm{E}=16.5 \mathrm{keV})$, having beam dimensions of approximately $200 \times 400 \mu \mathrm{m}$, and had a typical 30 flux of $10^{13}$ photons $/ \mathrm{s}$. The distance between the sample and detector was set at $1.5 \mathrm{~m}$, and the 2-D powder diffraction patterns were recorded on an image-intensified FReLoN Kodak CCD detector with an input field of $100 \times 100 \mathrm{~mm}$. Silver behenate $(\mathrm{a}=58.38 \AA)$ was used to calibrate the low35 angle x-ray diffraction for all measurements. Diffraction images were analysed by the IDL-based AXcess software package, developed in-house by Dr. A. Heron ${ }^{29}$. The measured $\mathrm{x}$-ray spacings are accurate to $\pm 0.1 \AA$. The program CrystalMaker ${ }^{\circledR}$ (CrystalMaker Software Ltd, Oxford, England 40 - www.crystalmaker.com) was used to visualize 2-D sections through the model structure of the Fd3m cubic phase.

\section{Results and Discussion}

\subsection{X-ray data and electron density maps}

${ }_{45}$ As mentioned in the Introduction, fully hydrated unsaturated diacylglycerol/ phosphatidylcholine mixtures are found to adopt an inverse micellar cubic phase, of crystallographic space group $\mathrm{Fd} 3 \mathrm{~m}$. In the present study, hydrated DOPC: DOG mixtures with a molar ratio close to $1: 2$ were examined 50 over a large pressure range, at a number of temperatures, using synchrotron small-angle $\mathrm{x}$-ray diffraction. A typical diffraction pattern of the $\mathrm{Fd} 3 \mathrm{~m}$ phase obtained at $16{ }^{\circ} \mathrm{C}$ and 1 bar is shown in Fig. 3a. The quality of the data is much higher than those previously obtained using laboratory X-ray ${ }_{55}$ sources. A radial integration about the centre of the pattern is presented in Fig. 3b, showing the intensities of the individual Bragg reflections, along with their assigned Miller indices. A total of 13 reflections are observed, which index as the 111 , 220, 311, 222, 400, 331, 422, $333 / 511,440,531,620,533$ 95 and 622 reflections of a cubic phase of cubic aspect 15 $(\mathrm{Fd} 3 \mathrm{~m} / \mathrm{Fd} 3)$, assumed to be spacegroup $\mathrm{Fd} 3 \mathrm{~m}^{30-31}$, with a lattice parameter of $133.3 \pm 0.1 \AA$.

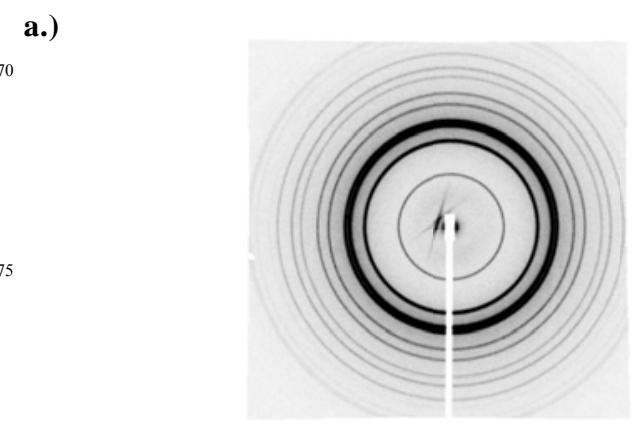

b.)

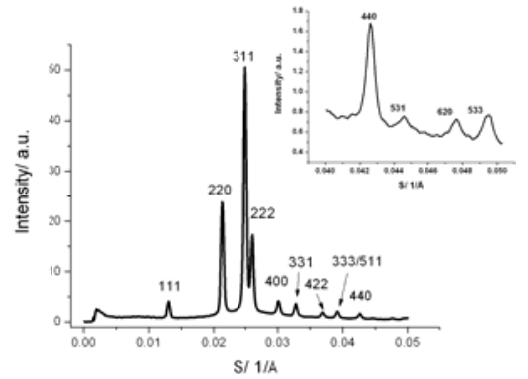

Fig. 3 (a) Low-angle synchrotron X-ray diffraction pattern from the Fd3m cubic phase of hydrated DOPC/DOG; (b) the integrated 1-D pattern, 110 along with the indexing of the Bragg peaks. The inset shows the zoomedin region of the integrated 1-D pattern beyond peak 440.

Based on our previous data of lattice parameter versus water content $^{2}$, we estimate the water content of the sample to be 145 close to $19 \mathrm{wt} \%$, or slightly below limiting hydration. The indexing of the diffraction datat is shown in Fig. 4, as a plot of the reciprocal $d$-spacings $\left(1 / \mathrm{d}_{\mathrm{hkl}}\right)$ versus $m=\sqrt{ }\left(\mathrm{h}^{2}+\mathrm{k}^{2}+\mathrm{l}^{2}\right)$. For a correctly-indexed cubic phase, such a plot should be linear, with a gradient equal to the reciprocal of the lattice parameter 150 of the phase, and should pass through the origin. The fit is excellent, and the gradient of the line yields the lattice parameter quoted above.

The relative intensity for each of the 13 Bragg peaks along with their Miller indices is listed in Table 1. The observed 130 intensities, corrected by a factor of $q^{2}$ (Lorentz correction) $)^{32}$, to take account of the SAXS geometry, are scaled as percentages of the most intense (311) reflection.

The observed intensities were used to construct an electron density map using the approach previously described by 140 Luzzati and co-workers ${ }^{3,31,33-34}$.The Lorentz corrected, normalised intensities were corrected by the appropriate multiplicity factor for each reflection, and converted to structure factor moduli $\left(\mathrm{IF}_{\mathrm{hkl}} \mathrm{I}\right)$ (Table 1). ). The overlapping 
$333 / 511$ reflections have been assumed to have equal values of intensity ${ }^{31,33}$. The electron density map was computed by Fourier summation using the phasing of the first ten structure factors previously determined by Luzzati et al., ${ }^{3}$ and the 5 phases of the remaining three diffraction orders were determined by trial and error (Table 1). As the intensities of these last three diffraction orders are very weak, changing the phase of the structure factors only gave rise to subtle differences in the electron density maps. The 2-D sections of 10 the resulting electron density map are shown in Fig. 5

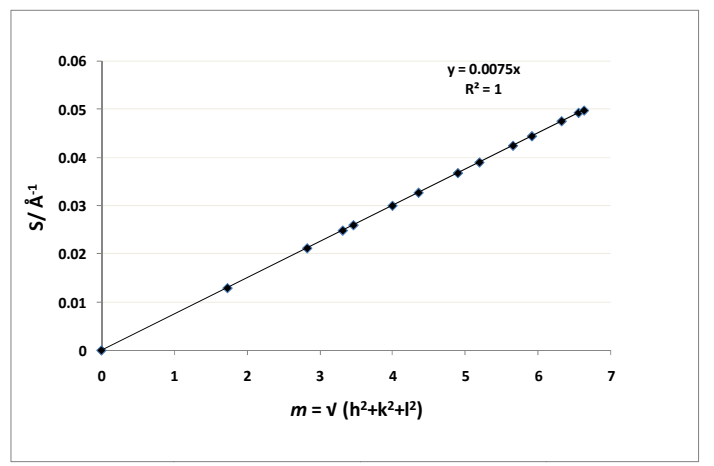

Fig. 4 The observed reciprocal spacings versus $v\left(h^{2}+k^{2}+l^{2}\right)$ fit perfectly to a linear plot through the origin, confirming the indexing as cubic spacegroup Fd3m

15

\begin{tabular}{|c|c|c|c|c|c|}
\hline $\begin{array}{c}\text { Experimental } \\
\text { d-spacings / } \\
\AA\end{array}$ & $\begin{array}{c}\text { Miller } \\
\text { indices }\end{array}$ & $\begin{array}{c}\text { Intensity } \\
\mathrm{I}(\mathrm{q}) \mathrm{q}^{2}\end{array}$ & Multiplicity & $\mathrm{IF}_{\mathrm{hkl}} \mathrm{I}$ & Phase \\
\hline 77.3 & 111 & 1.7 & 8 & 0.119 & - \\
\hline 47.2 & 220 & 33.1 & 12 & 0.430 & - \\
\hline 40.2 & 311 & 100 & 24 & 0.529 & - \\
\hline 38.5 & 222 & 31.7 & 8 & 0.516 & + \\
\hline 33.3 & 400 & 9.7 & 6 & 0.329 & + \\
\hline 30.5 & 331 & 9.3 & 24 & 0.161 & - \\
\hline 27.2 & 422 & 5.5 & 24 & 0.124 & - \\
\hline 25.6 & 333 & 3.9 & 8 & 0.180 & - \\
\hline 25.6 & 511 & 3.9 & 24 & 0.104 & - \\
\hline 23.5 & 440 & 6.7 & 12 & 0.193 & - \\
\hline 22.5 & 531 & 1.4 & 48 & 0.044 & - \\
\hline 21.0 & 620 & 1.5 & 24 & 0.065 & - \\
\hline 20.3 & 533 & 3.1 & 24 & 0.093 & + \\
\hline
\end{tabular}

Table 1. The experimental X-ray spacings, intensities and moduli of the structure factors, along with the phasing used for the electron density maps.
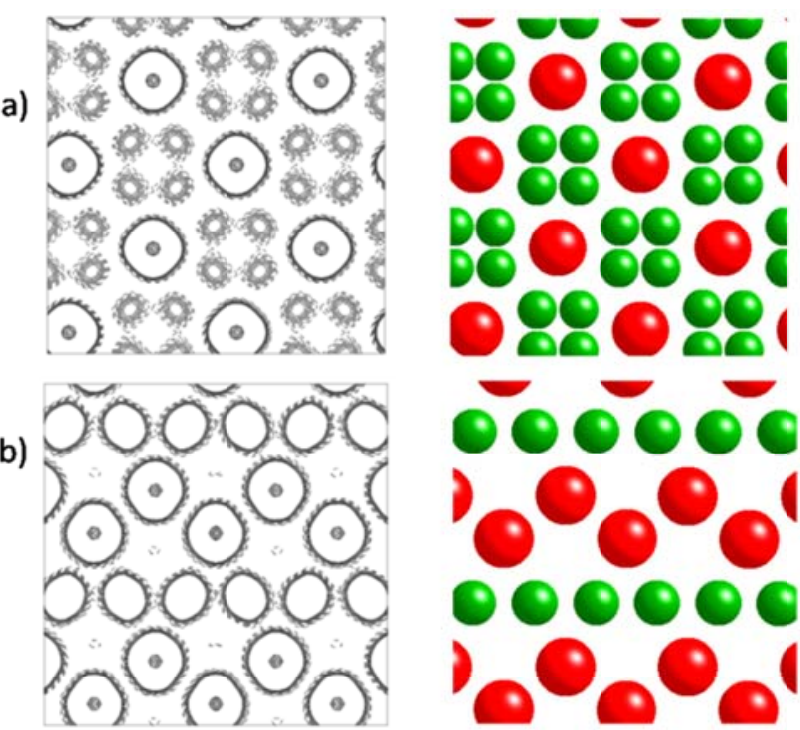

c)
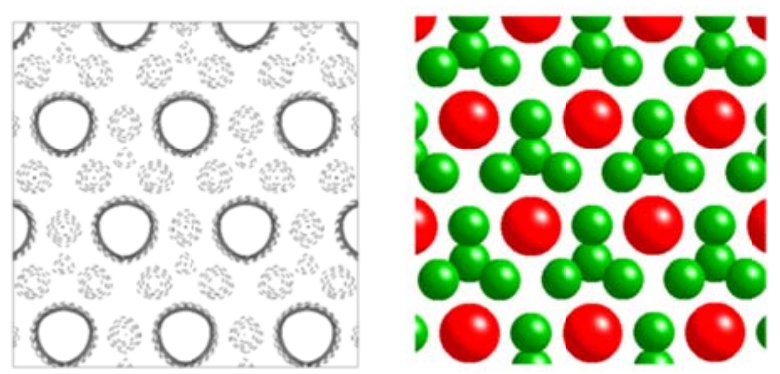

Fig.5 2-D sections through the electron density map (four unit cells are shown), along with corresponding sections visualized using CrystalMaker software, through the schematic structure shown in Fig 1. (a) (001) 100 section (symmetry $\mathrm{p} 4 \mathrm{~mm}$ ) through $\mathrm{z}=\mathrm{a} / 8$. The unit cell origin is at the bottom left corner. Note that half of the green micelles are slightly above, and half slightly below, the section through the centres of the red micelles; (b) 110 section (symmetry c $2 \mathrm{~mm}$ ) through (5a/8, 5a/8, z); (c) (111) section (symmetry $\mathrm{p} 6 \mathrm{~mm}$ ) through $(7 \mathrm{a} / 8,7 \mathrm{a} / 8,7 \mathrm{a} / 8)$. Note that 105 three of the green micelles are above, and the central one below, the section through the centres of the red micelles. Fd3m origin 2 (at $\overline{3} \mathrm{~m}$ ) is used in these sections; this is displaced from origin 1 by $(a / 8, a / 8, a / 8)$ (see Fig. 1).Note also that the sections shown do not encompass the whole unit cell and thus do not appear to show the full symmetries of the 001,110 and 111 projections.

By examining the sections it can be seen that the $\mathrm{Fd} 3 \mathrm{~m}$ phase consists of quasi-spherical inverse micellar aggregates of two different types, 8 large and 16 small per unit cell. From these 120 electron density sections we estimate that the polar cores of the two types of inverse micelle have diameters of roughly $0.344 \mathrm{a}=46 \AA$, and $0.266 \mathrm{a}=35 \AA$, respectively, based on the measured lattice parameter of $\mathrm{a}=133 \AA$. This compares quite well with our previous estimates of 56 and $44 \AA$ for the polar ${ }_{125}$ core diameters of an oleic acid / sodium oleate $\mathrm{Fd} 3 \mathrm{~m}$ phase ${ }^{2}$, with a larger lattice parameter of $150 \AA$. One must be rather careful in interpreting such sections from electron density maps. In certain 2-D sections (data not shown), the smaller inverse micelles can appear larger than the actual larger ones, 130 if the section cuts through their centres, but is offset from the centres of the larger ones.

We hypothesise that the DOPC will preferentially partition 
into the larger, less curved micelles and vice versa for the less hydrophilic DOG component, based on their relative packing parameters / spontaneous curvatures. Isomorphous replacement or neutron diffraction contrast variation 5 experiments could be carried out to confirm this, and this is currently being undertaken. A variety of experimental techniques have shown that DAGs form a hydrogen bond between the DAG hydroxyl group and the phospholipid ester group resulting in elimination of bound water from the 10 phospholipid headgroup causing surface dehydration ${ }^{19,35}$. This interaction of the two species prevents phase separation within the $\mathrm{Fd} 3 \mathrm{~m}$ phase but does not stop segregation into the two types of micelle.

The packing fraction, defined as the percentage of the unit cell 15 volume occupied by the micelles, for the Fd3m clathrate structure is 0.71 . However, such packing fractions are only relevant for hard spheres, as fluid interfaces may be deformed (this is known for the $\mathrm{Fd} 3 \mathrm{~m}$ phase) potentially producing a packing fraction approaching $100 \%$. This deformation can be 20 seen in the electron density maps as the interfaces of the inverse micelles are clearly faceted.

\subsection{Hydrostatic pressure effects on DOPC:DOG $\left(c_{\text {DOG }}=0.7\right)$ mixtures}

${ }_{25}$ The pressure dependence of the phase behaviour and lattice parameters of the hydrated DOPC:DOG system at selected temperatures has been investigated. Although many studies have investigated the pressure dependence of the lamellar, hexagonal and bicontinuous cubic phases, only a small 30 number of studies have investigated the pressure dependence of the $F d 3 m$ phase ${ }^{10,26}$. Fig. 6 shows the effect of pressure on the phase stability and phase structure of this hydrated DOPC:DOG mixture at $30^{\circ} \mathrm{C}$, which is initially in the $\mathrm{Fd} 3 \mathrm{~m}$ cubic phase at atmospheric pressure.

${ }_{35}$ There are a number of very striking observations: Firstly, a number of structural transitions are induced, first by increasing pressure, and then by decreasing pressure. It is clear that the transitions occur sharply, at well-defined pressures, and are reversible, although with some apparent 40 hysteresis (for example, the transition out of the Fd3m cubic phase occurs at 1,900 bar on increasing pressure, but the phase only reforms when the pressure is reduced below approx 600 bar).

Secondly, the transitions occur from a single $(\mathrm{Fd} 3 \mathrm{~m})$ phase to 45 a pair of coexisting phases. Thus the pure $\mathrm{Fd} 3 \mathrm{~m}$ phase transforms to a coexisting inverse hexagonal $\left(\mathrm{H}_{\mathrm{II}}\right)$ and lamellar phase (believed to be an ordered lamellar phase, denoted an $\mathrm{L}_{\mathrm{c}}$ phase) at 1900 bar. The $\mathrm{H}_{\text {II }}$ phase then converts into a second lamellar phase (believed to be a fluid lamellar

${ }_{50} \mathrm{~L}_{\alpha}$ phase) at 2900 bar, coexisting with the $\mathrm{L}_{c}$ phase. The clear implication of this phase separation is that, since DAGs are known to be immiscible with phospholipids at low temperatures $^{19,36}$ (and high pressures), the phase separated mixture at $3 \mathrm{kbar}$ most likely consists of a DOG-rich ordered 55 lamellar $\mathrm{L}_{\mathrm{c}}$ phase (lattice parameter $\approx 38 \AA$ ), and a DOG-poor fluid lamellar $\mathrm{L}_{\alpha}$ phase (lattice parameter $\approx 65 \AA$ ). The DOGrich phase is assumed to be an ordered lamellar phase since at this temperature and pressure it should be below its melting transition temperature $\left(\approx 25^{0} \mathrm{I}\right.$ at atmospheric pressure), as 90 we have measured by DSC. DOPC is known to adopt a fluid lamellar phase $\left(\mathrm{L}_{\alpha}\right)$ at all temperatures and pressures investigated here ${ }^{27,37}$ and so we infer that the DOPC-rich phase is a fluid lamellar $\left(\mathrm{L}_{\alpha}\right)$ phase.

Upon reducing the pressure, first the fluid lamellar phase 115 transforms to the $\mathrm{H}_{\text {II }}$ phase, then at a much lower pressure both the $\mathrm{H}_{\mathrm{II}}$ phase and the coexisting ordered lamellar phase simultaneously transform back into a single pure Fd3m cubic phase. With the experimental set-up at beamline ID02, we were not able to prove these conclusions fully, as we could 120 not measure the wide-angle diffraction, which would confirm the packing state of the chains (fluid, gel, or more-highly ordered). However, the observed spacings from the low-angle patterns are fully consistent with our conclusions.

Thirdly, the lattice parameter of the $\mathrm{Fd} 3 \mathrm{~m}$ cubic phase is 105 practically unchanged upon raising the pressure from 1 bar 2,000 bar [Fig. 7]. This behaviiour is quite different from that observed for inverse bicontinuous cubic phases in excess water, where the lattice parameter increases - sometimes very significantly - with increasing pressure ${ }^{26,29,38}$. 85

(a)

90

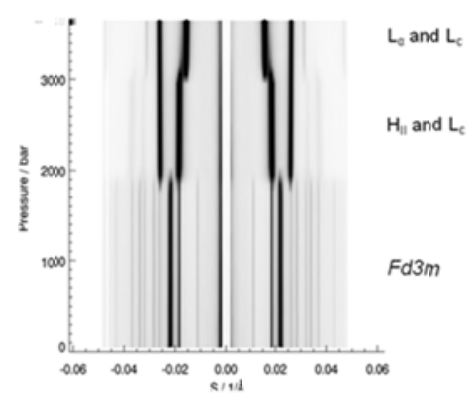

(b) 100

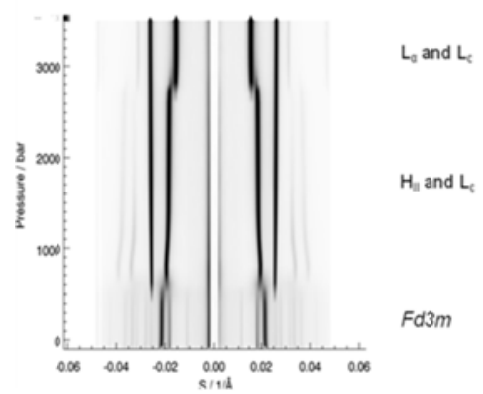

Fig. 6 Phase transitions induced in hydrated DOPC/DOG at $30{ }^{\circ} \mathrm{C}$ by varying the hydrostatic pressure: (a) between 1 bar and $3.5 \mathrm{kbar}$ (upwards, pressurisation), and (b) between 3.5 kbar and 1 bar (downwards, depressurisation).

Although previous studies on glycolipids ${ }^{26}$ did not show any clear trend on the effect of pressure on the lattice parameter of the $\mathrm{Fd} 3 \mathrm{~m}$ phase, a recent study by Yaghmur et al., on the 45 effect of pressure on the $\mathrm{Fd} 3 \mathrm{~m}$ phase formed by monoolein: tetradecane mixtures showed a large pressure induced swelling of the $\mathrm{Fd} 3 \mathrm{~m}$ phase $\mathrm{e}^{10}$. The difference in this system from ours is that upon pressurizing, the extra alkane present 
can be incorporated in the lipid phase and therefore swell it, whereas for our DOPC:DOG system this cannot occur. For the water cores of inverse micellar ordered phases to swell would require water molecules to penetrate the highly hydrophobic 5 acyl chains region which would be kinetically very slow; furthermore, the chain packing frustration would rise very rapidly as the polar cores increased in size. The consequence of this is that pressure has no effect on the lattice parameter for the DOPC:DOG Fd3m cubic phase.

10

15

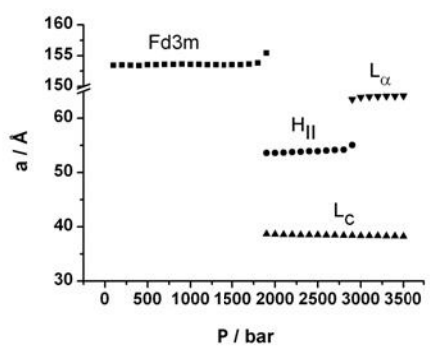

20

Fig. 7 The observed lattice parameters of the various lyotropic mesophases versus pressure.

\subsection{Temperature effects}

${ }_{25}$ The phase transitions for the DOPC:DOG mixture as a function of temperature at 1 bar are shown in Fig. 8 and correlate well with the pressure dependent results.

30

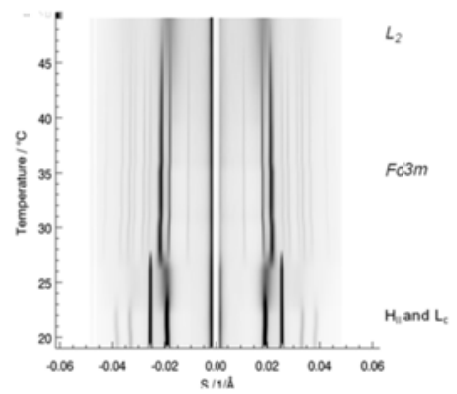

Fig 8. Phase transitions induced by varying temperature between $19^{\circ} \mathrm{C}$ 40 and $49^{\circ} \mathrm{C}$ in the upwards heating regime.

It is clearly seen that the coexisting ordered lamellar $\mathrm{L}_{c}$ and $\mathrm{H}_{\text {II }}$ phases transform into the pure $\mathrm{Fd} 3 \mathrm{~m}$ phase at approx 27

${ }^{0} \mathrm{C}$, which in turn starts to convert into the $\mathrm{L}_{2}$ inverse micellar 45 solution phase at $47{ }^{\circ} \mathrm{C}$. Interestingly, there is some evidence (Fig. 8) of diffuse scatter around the first order (10) peak of the $\mathrm{H}_{\text {II }}$ phase, just prior to the transition to the $\mathrm{Fd} 3 \mathrm{~m}$ phase. This suggests that the transition mechanism first involves a disordering of the (fluid) $\mathrm{H}_{\mathrm{II}}$ phase, before the ordered so lamellar phase disappears (melts), and the Fd3m cubic phase forms.

The $\mathrm{Fd} 3 \mathrm{~m}-\mathrm{L}_{2}$ transition is not sharp, and is found to proceed via a coexistence region. Moreover, we find that the reverse $\mathrm{L}_{2}-\mathrm{Fd} 3 \mathrm{~m}$ transition in the cooling regime shows hysteresis,
140 and has an even broader coexistence region (data not shown). This may be due to the kinetics of transformation, which on heating should be fast, as the Fd3m micelles only have to lose their ordered packing in order to transform to the inverse micellar solution, which presumably consists of inverse 145 micelles with some degree of size distribution. We expect however that the kinetics in the cooling region should be much slower, since the phase change would require a large scale rearrangement of water. Furthermore, the lipid species would also have to partially phase separate in order to form 150 the two different sized micelles in the $\mathrm{Fd} 3 \mathrm{~m}$ phase, and then the micelles would finally have to come together in the correct $2: 1$ ratio of smaller:larger size, and then pack into the ordered lattice. Slow kinetics of formation of the $\mathrm{Fd} 3 \mathrm{~m}$ phase from an $\mathrm{L}_{2}$ solution has indeed been observed ${ }^{6,12}$. Unfortunately our 155 system does not have a sharp $\mathrm{Fd} 3 \mathrm{~m}-\mathrm{L}_{2}$ transition that would allows us to investigate the kinetics of the transition.

\section{Conclusions and future directions}

Ordered inverse micellar cubic phases formed by hydrated lipid mixtures show a number of striking features when 125 subjected to hydrostatic pressure, and behave quite differently from bicontinuous inverse cubic phases. It will be of considerable interest to carry out pressure-jump time-resolved $\mathrm{X}$-ray diffraction measurements on such systems in order to probe the kinetics and mechanisms of the various phase 130 transitions and phase separations involved. To be able to do this will require a more detailedl knowledge of the pressuretemperature phase diagram.

Dedication We would like to dedicate this article to 105 Professor Otto Glatter on the occasion of his $65^{\text {th }}$ birthday, in recognition of his seminal contributions, both to scattering and to soft matter self-assembly.

Acknowledgements We thank the EPSRC (UK) for Platform grant EP/G00465X, and for a Chemical Biology DTC 120 Studentship to AIIT. We thank the ESRF for the award of synchrotron beamtime, Dr. T. Narayanan, Dr. M. Sztucki and other staff on beamline ID02 for their support and assistance, and Professor R. Winter for access to the Dortmund pressure cell.

\section{Notes and references}

$110{ }^{a}$ Membrane Biophysics Platform and Institute of Chemical Biology, Department of Chemistry, Imperial College London, South Kensington Campus, London SW7 2AZ, UK.

${ }^{b}$ University Paris Sud 11, F-91405 Orsiay cedex, France

* j.seddon@imperial.ac.uk; r.law@imperial.ac.uk

105

1 G.C. Shearman, O. Ces, R.H. Templer and J.M. Seddon, J. Phys.: Condens. Matter, 2006, 18, S1105-S1124

2 J.M. Seddon, J. Robins, T. Gulik-Krzywicki and H. Delacroix, Phys. Chem. Chem. Phys., 2000, 2, 4485-4493

1153 V. Luzzati, R. Vargas, A. Gulik. P. Mariani, J.M. Seddon and E. Rivas, Biochemistry, 1992, 31, 279-285

4 Z. Huang, J.M. Seddon and R.H. Templer, Chem. Phys. Lipids, 1996, 82, 53-6.

5 J.M. Seddon, Biochemistry, 1990, 29, 7997-8002 
6 M. Minamikawa, and M. Hato, Langmuir, 1998, 14, 4503-4509

7 J.M. Seddon, N. Zeb, R.H. Templer, R.N. McElhaney and D.A. Mannock, Langmuir, 1996, 12, 5250-5253

8 S. Guillot, C. Moitzi, S. Salentinig, L. Sagalowicz, M.E. Leser and O. Glatter, Colloids Surf., A, 2006, 291, 78-84

9 A. Yaghmur, L. de Campo, S. Salentinig, L. Sagalowicz, M.E. Leser and O. Glatter, Langmuir, 2006, 22, 517-521

10 A. Yaghmur, M. Kriechbaum, H. Amenitsch, M. Steinhart, P. Laggner and M. Rappolt, Langmuir, 2010, 26, 1177-1185.

1011 G.C. Shearman, B.J. Khoo, M.L. Motherwell, K.A. Brakkle, O.Ces, C.E. Conn, J.M. Seddon and R.H. Templer, Langmuir, 2007, 23, 7276-7285

12 M. Pouzot, R. Mezzenga, M. Leser, L. Sagalowicz, S. Guillot and O. Glatter, Langmuir, 2007, 23, 9618-9628.

1513 A. Salonen, S. Guillot, and O. Glatter, Langmuir, 2007, 23, 91519154.

14 S. Guillot, S. Salentinig, A. Chemelli, L. Sagalowicz, M.E. Leser and O. Glatter, Langmuir, 2010, 26, 6222-6229

15 J.C. Gomez-Fernandez and S. Corbalan-Garcia, S., Chem. Phys. Lipids, 2007, 148, 1-25

16 M.H. Fukami and H. Holmsen, Eur. J. Biochem., 1995, 228, 579-586

17 G.C.Shearman, A. I. I. Tyler, N. J. Brooks, R. H. Templer, O. Ces, R. V. Law, J. M. Seddon, J. Am. Chem. Soc. 131, 1678-1679

18 S. A. El-Safty, A.A. Ismail, H. Matsunaga, H. Nanjo and F. Mizukami, 25 J. Phys. Chem. C, 2008, 112, 4825-4835

19 F. Lopez-Garcia, J. Villalain and P.J. Quinn, Biophys. J., 1994, 66, 1991-2004

20 A. Jimenez-Monreal, J. Villalain, F.J. Aranda and J.C. GomezFernandez, Biochim. Biophys. Acta, 1998, 1373, 209-219

3021 T. Heimburg, U. Wurz and D. Marsh, Biophys. J., 1992, 63, 13691378

22 S. Smith, I. Kustanovich, S. Bhamidipati, A. Salmon and J.A. Hamilton, Biochemistry, 1992, 31, 11660-11664

23 P. Quinn, H. Takahashi and I. Hatta, Biophys. J., 1995, 68, 1374 1382

24 J. Nieva, A. Alosno, G.Basanez, F.M. Goni, A. Gulik, R. Vargas and V. Luzzati, FEBS lett., 1995, 368, $143-147$

25 D. Armstrong, D.B. Borchardt and R. Zidovetzki, Biochem. Biophys. Res. Commun., 2002, 396, 806-812

4026 P.M. Duesing, J.M. Seddon, R.H. Templer and D.A. Mannock, Langmuir, 1997, 13, 2655-2664

27 R. Winter, Biochim. Biophys. Acta, 2002, 1595, 160-184

28 J. Woenckhaus, R. Kohling, R. Winter, P. Thiyagarajan and S. Finet, S., Rev. Sci. Instrum., 2000, 71, 3895-3899

4529 J.M. Seddon, A.M. Squires, O. Ces, A.J. Heron, X. Mulet, G.C. Shearman and R.H. Templer, Philos. Trans. R. Soc. A, 2006, 364, 2635-2655

30 Hahn, T., Ed. (1983) International Tables for Crystallography, Vol. A, D. Reidel, Dordrecht, Holland

5031 P. Mariani, E. Rivas, V. Luzzati and H. Delacroix, Biochemistry, 1990, 29, 6799-6810

32 Janssen T., Janner A., Looijenga-Vos A., de Wolff P. M., International Tables for Crystallography; Kluwer: London, UK, 1995

5533 Mariani, P., Luzzati, V., and Delacroix, H., J. Mol. Biol., 1988, 204, 165- 189

34 H. Delacroix, T. Gulik-Krzywicki and J.M. Seddon, J. Mol. Biol., 1996, 258, 88-103

35 E. Goldberg, D.S. Lester and R. Zidovetzki, Biophys. J., 1995, 69, 965-973

36 J.O. Hindenes, W. Nerdal, L. Di, D.M. Small and H Holmsen, J. Biol. Chem., 2000, 275, 6857-6867

37 B.A. Bergenstahl and P. Stenius, J. Phys. Chem., 1987, 91, 5944 5948

6538 R. Winter, J. Erbes, R.H. Templer, J.M. Seddon, A. Syrykh, N.A. Warrender and G. Rapp, Phys. Chem. Chem. Phys., 1999, 1, 887-893 